\title{
IHTC14-23115
}

\section{HEAT AND MASS TRANSPORT IN CARBON NANTUBES}

\author{
Junichiro Shiomi \\ Department of Mechanical \\ Engineering \\ The University of Tokyo \\ 7-3-1 Hongo, Bunkyo-ku, \\ Tokyo, 113-8656, Japan
}

\author{
Carl Fredrik Carlborg \\ Microsystem Technology \\ Laboratory, \\ Royal Institute of Technology, \\ SE-100 44 Stockholm, Sweden
}

\author{
Shigeo Maruyama \\ Department of Mechanical \\ Engineering \\ The University of Tokyo \\ 7-3-1 Hongo, Bunkyo-ku, \\ Tokyo, 113-8656, Japan
}

\begin{abstract}
We have investigated heat and mass transport in singlewalled carbon nanotubes (SWNTs) using molecular dynamics methods. Particular attention was paid on the non-equilibrium dynamics at the interface between SWNT and other materials, which strongly manifests in nanoscale. In the first part, we have investigated the heat transport through the interface between SWNTs and surrounding argon matrices in liquid and solid phases. By analyzing the energy relaxation from SWNT to the matrices using non-stationary molecular dynamics simulations, elastic and inelastic thermal energy transports across the interface were separately quantified. The result reveals that the elastic interaction transports energy much faster than the inelastic one, but carries much smaller energy due to slow intra-SWNT phonon relaxation. In the second part, we have investigated a possibility to utilize nonequilibrium thermal interface to transport water through an SWNT. By applying the longitudinal temperature gradient to the SWNT, it is demonstrated that the water cluster is efficiently driven at average acceleration proportional to the temperature gradient. However, the transport simulations with a junction of two different SWNTs suggest that an angstrom diameter difference may result in a significant drag for small diameter SWNTs.
\end{abstract}

\section{INTRODUCTION}

Carbon nanotubes are one of the key materials in nanotechnology owning to their extraordinary mechanical, thermal, electrical, and optical properties. In particular, singlewalled carbon nanotubes (SWNTs) with ideal quasi-onedimensional structure have caught much attention as a material realizing various low-dimensional transport properties [1]. While much of the earlier efforts in this field have been devoted into research on electrical transport, there are increasing interests in low dimensional heat and mass transports of carbon nanotubes that are beneficial for various applications.

Characterization of thermal properties of carbon nanotubes is a key issue for their prospective electrical and thermal device applications. The importance of probing carbon nanotubes heat conduction has attracted many recent researches, which have revealed remarkable heat conduction properties [2-7]. The heat conduction exhibits complex diffusive-ballistic feature for realistic nanotube-length in many applications even at room temperature. As a consequence, unique steady [8-13] and unsteady [14] heat conduction characteristics manifest. They are particularly evident in the length effect of thermal conductance, where heat conduction does not become fully diffusive even for SWNTs as long as a micrometer [15].

While these quasi-ballistic thermal transport properties encourages SWNTs for thermal/electrical device applications, on considering integrated systems in practical situations, interfacial heat transfer between nanotubes and surrounding materials becomes important. In many practical devices, the applicable heat load to the system is determined by the thermal boundary conductance (TBC) between SWNT and surrounding heat sinks/sources rather than the thermal conductance through a carbon nanotube itself. One representative case is the carbon nanotube composite, which has been explored widely aiming at bulk applications [16-19]. In this study, MD simulations were performed to probe the physics of TBC, which is confined in atomic-scale layers adjacent to the SWNT and strongly dependent on their local properties. Contributions of the elastic and inelastic scatterings on the $\mathrm{TBC}$ and its dependence on the matrix phase have been quantified by analyzing the vibrational spectra [20]. 
In addition to heat transport, mass transport could also benefit from the quasi-one-dimensional structure of carbon nanotubes. Since water intrusion into hydrophobic SWNTs has been demonstrated to be possible [21-23], possibilities of using SWNTs as molecular transporter and filters have been explored with merits of realizing fast transport even in nanoscale pores attributing to the exceptionally large slip-length at the hydrophobic and atomically smooth interface [24-27]. In this course, we have investigated the transport of a water cluster through an SWNT under longitudinal temperature gradient [28]. It is demonstrated that the water cluster is driven with an average acceleration proportional to the temperature gradient. On the other hand, the transport simulations with a junction of two different SWNTs suggest that an angstrom diameter difference may result in a significant drag for small diameter SWNTs.

\section{MOLECULAR DYNAMICS SIMULATIONS}

The carbon-carbon interactions were modeled using Brenner potential [29], where the total potential energy of the system is expressed as,

$$
E=\sum_{i} \sum_{j(i<j)}\left[V_{R}\left(r_{i j}\right)-B_{i j}^{*} V_{A}\left(r_{i j}\right)\right]
$$

Here, $V_{R}(r)$ and $V_{A}(r)$ are repulsive and attractive force terms, which take a Morse type form with a certain cut-off function. $B_{i j}^{*}$ represents the effect of the bonding order parameters. As for the potential parameters, we employ the set that has been shown to reproduce the linear phonon transport properties with sufficient accuracy [14]. The velocity Verlet method was adopted to integrate the equation of motion with the time step of $0.5 \mathrm{fs}$.

Water molecules were expressed by the SPC/E potential [30]. The SPC/E potential is expressed as the superposition of Lennard-Jones function of oxygen-oxygen interaction and the electrostatic potential by charges on oxygen and hydrogen as follows,

$$
\phi_{12}=4 \varepsilon_{\mathrm{OO}}\left[\left(\frac{\sigma_{\mathrm{OO}}}{R_{12}}\right)^{12}-\left(\frac{\sigma_{\mathrm{OO}}}{R_{12}}\right)^{6}\right]+\sum_{i} \sum_{j} \frac{q_{i} q_{j} e^{2}}{4 \pi \varepsilon_{0} r_{i j}},
$$

where $R_{12}$ represents the distance of oxygen atoms, and $\sigma_{O O}$ and $\varepsilon_{O O}$ are Lennard-Jones parameters. The Coulombic interaction is the sum of 9 pairs of point charges, where $r_{i j}$ denotes the distance between inter-molecular point charges. The potential function between water molecules and carbon atoms were represented by Lennard-Jones function of the distance between the oxygen in the water molecule and the

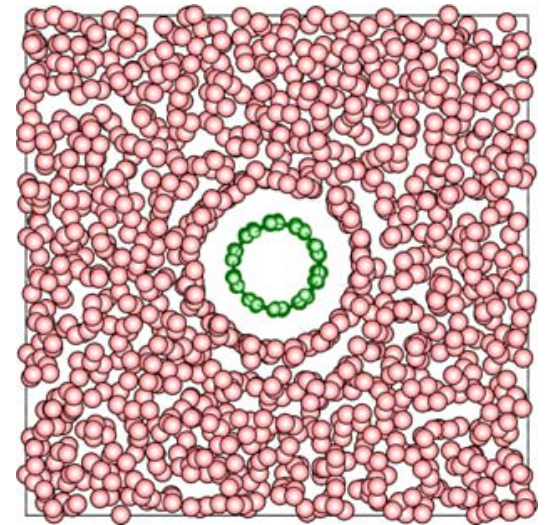

Fig. 1 SWNT surrounded by argon.

carbon atom. The parameters for the Lennard-Jones potential were $\varepsilon_{C O}=0.314 \mathrm{~kJ} / \mathrm{mol}$ and $\sigma_{C O}=3.18 \AA$.

\section{HEAT TRANSPORT FROM CARBON NANOTUBE TO SURROUNDIND MATERIAL}

\section{Carbon nanotube-argon system}

While the quasi-ballistic heat conduction of SWNTs [15] is attractive for thermal device applications, in practical situations, the thermal boundary resistance (or conductance) plays a major role in nanomaterials. The SWNT composite is a representative case where the overall thermal transport performance is determined by the thermal boundary conductance (TBC) between SWNT and the surrounding matrix. Here we present a study investigating the TBC between SWNT and argon (Ar) matrix (Fig. 1). By taking Ar as the matrix, which can be modeled accurately by simple potential models, TBC can be investigated for a wide range of parameters such as size and phase.

The potential functions are represented by the LennardJones potential function with parameters $\varepsilon_{A r-A r}=10.33 \mathrm{meV}, \sigma_{A r-}$ ${ }_{A r}=3.4 \AA, \varepsilon_{A r-C}=4.98 \mathrm{meV}, \sigma_{A r-A r}=3.38 \AA$. Simulations were conducted for armchair SWNTs with chirality $(5,5)$, which gives a diameter of $0.69 \mathrm{~nm}$. The cross sectional area of the simulation box was $3.5 \times 3.5 \mathrm{~nm}^{2}$ and periodic boundary conditions were applied in all directions. The carbon nanotube length was varied from 1 to $50 \mathrm{~nm}$. To generate solid and liquid phase matrices, argon atoms were packed in a face-centered cubic structure around an SWNT with densities of $3000 \mathrm{~kg} / \mathrm{m}^{3}$ and $1200 \mathrm{~kg} / \mathrm{m}^{3}$, respectively. Both systems were equilibrated at $300 \mathrm{~K}$ in supercritical phase and then cooled down at a rate of $1 \mathrm{~K} / \mathrm{ps}$ to their respective target temperatures, $40 \mathrm{~K}$ for the solid matrix and $120 \mathrm{~K}$ for the liquid matrix.

\section{Thermal boundary conductance}



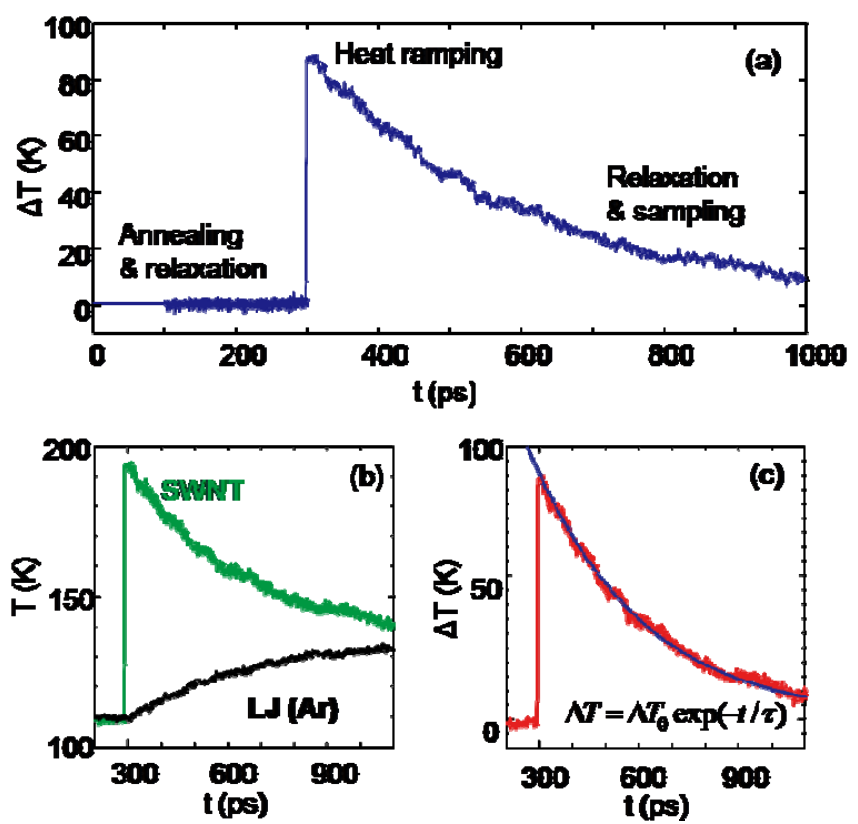

Fig. 2 (a) The different stages of the MD simulation. (b) After equilibration, the temperature of the SWNT is ramped up during $10 \mathrm{ps}$ and the system is relaxed to a new equilibrium without temperature control. (c) The temperature difference between the SWNT and the argon matrix is fitted to an exponential function, from which the relaxation time and the thermal boundary conductance can be calculated [20].

Figure 2 shows the complete temperature history during the simulations of a 22.5 -nm-long SWNT surrounded by a solid argon matrix. After equilibration, the matrices were disturbed out of equilibrium by ramping up the temperature of the SWNT by $200 \mathrm{~K}$ for $10 \mathrm{ps}$ and adiabatically relaxing them towards equilibrium without temperature control. During relaxation, the velocity components of the atoms were sampled and the temperature difference between the SWNT and the surrounding argon matrix was calculated. The temperature difference between the SWNT and the matrix during the relaxation can be well expressed by a single exponential function. Then the thermal boundary conductance $(K)$ can be determined using the lumped-heat capacity method as follows.

$$
\Delta T=\Delta T_{0} \exp \left[-\left(1 / m_{A r} c_{A r}+1 / m_{C N T} c_{C N T}\right) K S t\right]
$$

where $m$ and $c$ are mass and heat capacity of the material. The TBC of SWNT-matrix was calculated for nanotube-lengths $L$, ranging from 20 to $500 \AA$ and matrices of both solid and liquid phases. For both solid and liquid argon cases, $K$ is nearly constant at values of $2.4 \mathrm{MW} /\left(\mathrm{m}^{2} \mathrm{~K}^{-1}\right)$ at $40 \mathrm{~K}$ and 1.6 $\mathrm{MW} /\left(\mathrm{m}^{2} \mathrm{~K}^{-1}\right)$ at $120 \mathrm{~K}$, respectively, for the entire range of $L$ explored in the current study.

\section{Mode-dependent thermal energy transport}

The dynamics of the heat transfer can be studied based on the phonon energy spectrum of the nanotube, which is extracted by calculating the power spectral density of the velocity fluctuations

$$
g(f)=\frac{1}{N} \sum_{j}^{N}\left|\int \exp \left(-2 \pi i f t \mathbf{v}_{j} \mathrm{~d} t\right)\right|^{2}
$$

where $f$ is the frequency, $\boldsymbol{v}_{i}$ is the velocity vector of atom $j$ and $N$ is the number of atoms. The phonon spectrum for the solid argon matrix was calculated similarly at the interface, but only for argon atoms surrounding the SWNT. For the liquid argon matrix, the vibrational spectrum was obtained through the autocorrelation function of the velocity components for the argon atoms in the absorption layer. The argon adsorption layer was defined as a cylindrical control volume with the surface 4 $\AA$ from the SWNT surface.

Here, we present results in a temperature range from $40 \mathrm{~K}$ to $310 \mathrm{~K}$. The lower temperatures are low enough to severely violate the quantum limit of a carbon nanotube, bellow which the reduction of the heat capacity is significant. Thus, the current model system serves to highlight the classical molecular dynamics of the heat conduction.

The spectral analysis demonstrates linear coupling between the phonons in the SWNT and the vibrations of the adsorbed argon layer both in the liquid and solid argon matrices. In Fig. 3 , it can be seen that the lower frequency phonons of SWNT are attenuated by the surrounding argon matrix. This frequency range corresponds to the vibrational spectrum of the adjacent argon layer, which indicates elastic coupling between the SWNT and the argon. It is interesting to see that such modal coupling is observed not only for solid argon but for liquid argon. This can be attributed to the solid-like behavior of the high density argon adsorption layer.

When the SWNT is momentarily heated, SWNT goes through nonequilibrium states before relaxing to equilibrium. The nonequilibrium energy distribution depends on the modedependent intrinsic and interfacial phonon scatterings and transmission. Here, we express the nonequilibrium energy distribution of the SWNT by introducing the spectral temperature $T_{s p}(f, t)$, which is defined as

$$
T_{s p}(f, t)=\frac{g^{n e q}(f, t)}{g^{e q}(f)} T^{e q}
$$

Therefore, $T_{\mathrm{sp}}(f, t)$ can be obtained by calculating $g^{\text {neq }}$ and $g^{e q}$ separately by using equilibrium and non-equilibrium molecular dynamics simulations, respectively.

We now split the frequency range into the two regimes (1) $f=\left[0, f_{A r}\right]$, where the spectra of SWNT and argon overlaps, and (2) $f=\left[f_{A r}, f_{S W N T}\right]$, where they do not overlap. Here, $f_{A r}$ and $f_{S W N T}$ are the cutoff frequencies of SWNT and the adjacent argon layer. Then, the average spectral temperatures in the frequency regimes (1) and (2) can be expressed as, 


$$
\begin{aligned}
& T_{s p}^{(1)}(t)=\frac{T^{e q}}{f_{A r}} \int_{0}^{f_{A r}} \frac{g^{n e q}(f, t)}{g^{e q}(f)} d f, \\
& T_{s p}^{(2)}(t)=\frac{T^{e q}}{f_{\max }-f_{c}} \int_{f_{c}}^{f_{\max }} \frac{g^{\text {neq }}(f, t)}{g^{e q}(f)} d f
\end{aligned}
$$

The SWNT was given a heat pulse $(\Delta T=200 \mathrm{~K}, \Delta T=10 \mathrm{ps})$ and the spectral temperature, along with the average kinetic temperature of the argon matrix, were calculated during the relaxation to a new equilibrium. As shown in Fig. 4, for both matrices, $T_{s p}^{(1)}$ relaxes to the argon matrix temperature much faster than $T_{s p}^{(2)}$ (by factor 30 for solid argon and 8 for liquid argon). This implies that the elastic energy transport through the SWNT/argon interface is much faster than the inelastic one. This results in, as seen in Fig. 4, a strongly nonequilibrium phonon distribution in SWNT, where the low frequency modes are much colder than the high frequency ones. On the other hand, when comparing the amount of energy carried, the transported energy associated with the elastic transport channel amounts for less than $1 \%$ of the total heat transported to the argon, even for the solid matrix.

Figure 4 shows that both $T_{s p}^{(1)}$ and $T_{s p}^{(2)}$ are well expressed by single exponential function suggesting that both are single timescale relaxations. There should also be another timescale corresponding to the inter-mode energy relaxation of SWNT due to the finite thermal resistance of the SWNT, however, it was not observed in the current analyses. This suggests that the SWNT inter-mode energy relaxation is either much slower or faster than the current analyzed time window. The former scenario may be more likely on considering the long mean free paths and thus slow relaxation of low frequency phonons in SWNTs [15], which limits the number of collisions and thus the inter-mode energy transfer. Such slow inter-mode energy transfer may be particularly pronounced in the current system, where low frequency modes of the SWNT are severely attenuated. One may also suspect that this inter-mode energy transfer determines the timescale of $\mathrm{TBC}$, i.e. the single exponential relaxation (Fig. 4) is caused by the intrinsic intermode energy transfer of the SWNT instead of the interfacial energy transfer. However, that does not explain the observed density effect, where TBC increases with density.

The current result shows that the TBC from an SWNT to
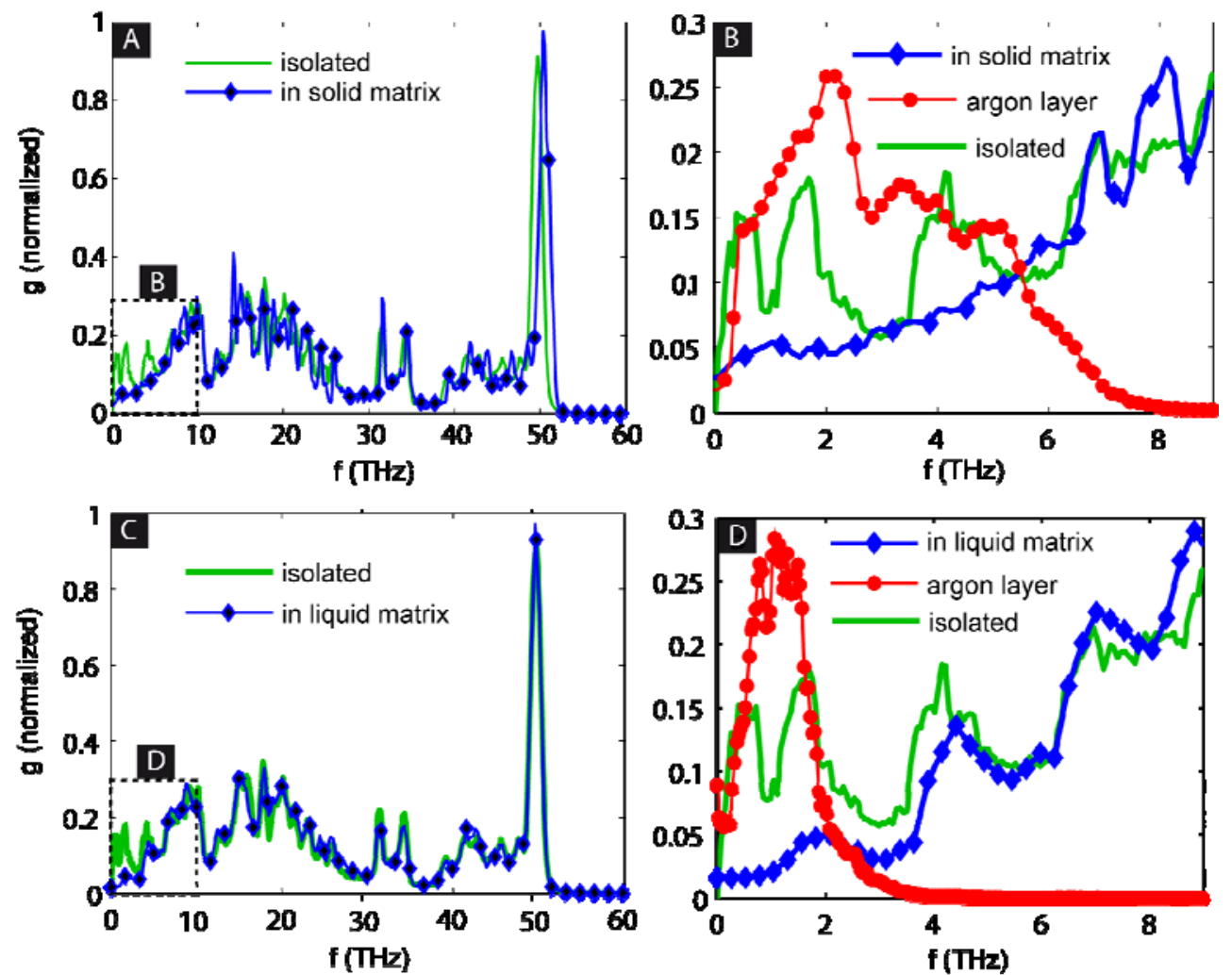

Fig. 3 (A) The full phonon spectrum of the $22.5 \mathrm{~nm}(5,5)$ SWNT submerged in the solid argon matrix (blue line, diamonds), compared to an isolated SWNT at the same temperature (green line). (B) A zoom-in on the first $9 \mathrm{THz}$ together with the phonon spectrum of the adjacent solid argon layer (red line, circles). (C) The full phonon spectrum of the SWNT in the liquid argon matrix compared to the isolated SWNT at the same temperature. (D) A zoom-in of the first $9 \mathrm{THz}$ compared with the vibrational spectrum of the adsorbed argon atoms [21]. 

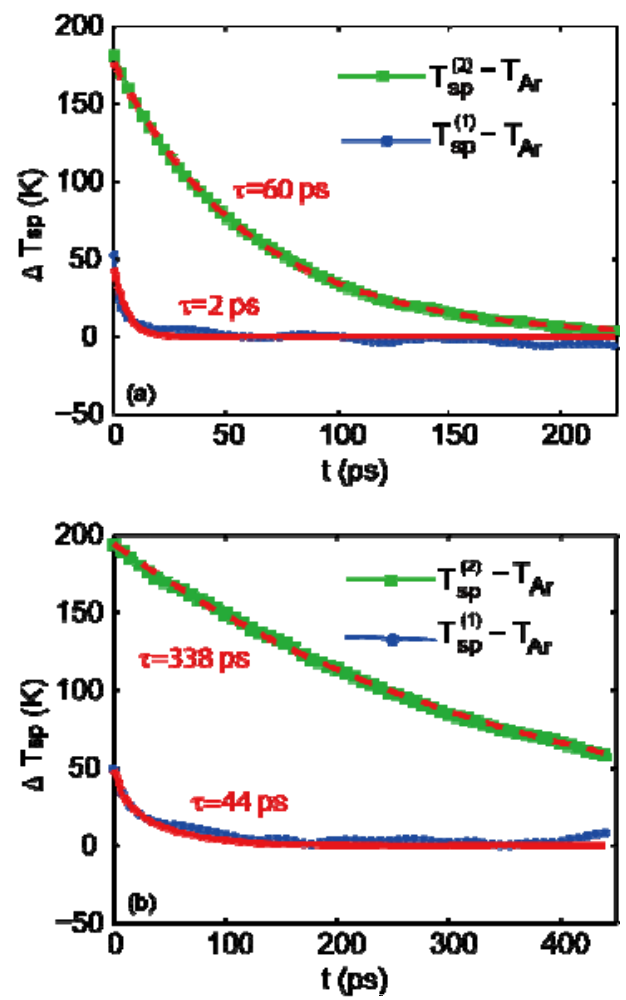

Fig. 4 Relaxations of spectral temperatures $T_{s p}^{(1)}$ and $T_{s p}^{(2)}$ of two frequency ranges $\left[0, f_{A r}\right]$ and $\left[f_{A r}, f_{S W N T}\right]$, respectively, for (a) solid and (b) liquid matrices (Ar) [21].

surrounding argon can be divided into two contributions; faster but smaller elastic energy transport and slower but larger inelastic energy transport. In the current classical system, the impact of the fast elastic energy transport appears minute, but it may become larger if we consider quantum effects with a higher population of lower frequency modes.

\section{WATER TRANSPORT INSIDE CARBON NANOTUBES DIRVEN BY TEMPEATURE GRADIENT}

\section{Methodology}

As shown in Fig. 5, the simulated system consists of a water cluster of 192 molecules confined in a $64 \mathrm{~nm}$ long (10,
10) SWNT with a diameter of $1.38 \mathrm{~nm}$. After initially equilibrating the system at around $300 \mathrm{~K}$, each end of the SWNT was cooled/heated by using Langevin thermostat to impose a temperature gradient along the nanotube with an average temperature of $300 \mathrm{~K}$. Until the quasi-steady temperature gradient is achieved, the mean axial translational velocity of the water cluster was cancelled to maintain the center of mass at $z=0$. Once the quasi-steady thermal gradient is achieved with constant heat flux through the SWNT, the water cluster was released and the position and velocity of the center of mass was recorded. The temperature difference $T_{h}-T_{c}$ was varied to investigate the sensitivity of the transport velocity to the temperature gradient.

\section{Water transport by temperature gradient}

The results of the above MD simulations clearly demonstrate the transport of water towards the cold end of the SWNT. The direction matches with the previous works on transport of sold nanoparticles [31,32]. Various phases of the transport are evident in the time history of the water cluster center of mass as shown in Fig. 6. After the initial phase, where the water cluster is pinned at $z=0$ (I), the water cluster accelerates at roughly constant acceleration (II) and, after a certain transient phase (III), eventually reaches a steady velocity (IV). For this case with a temperature gradient of 1.48 $\mathrm{K} / \mathrm{nm}$, the velocity reaches up to about $100 \mathrm{~m} / \mathrm{s}$. The water cluster eventually reaches the cold end and bounces back due to the increased potential outside the nanotube, but the thermal thrust keeps the cluster around the cold end (V). The time history of the mass center clearly reveals that the water cluster is driven with constant force which is balanced by the friction in the steady transport phase (IV). The friction is dominantly generated at the interface, judging from the observed plug-flow velocity distribution of the transported water.

The transport simulations were performed for various temperature gradients ranging from 0.18 to $1.48 \mathrm{~K} / \mathrm{nm}$. It is observed that the driving force increases proportionally with the temperature gradient. This suggests that the origin of the driving force lies in a quantity that has linear dependence on the temperature. One way to see this is in terms of the temperature dependence of the free energy. Since the free energy of the transported cluster depends on temperature, the state is expected to be led to more stable state, which is usually towards the cold side of the system. In a mind of enegetics, one

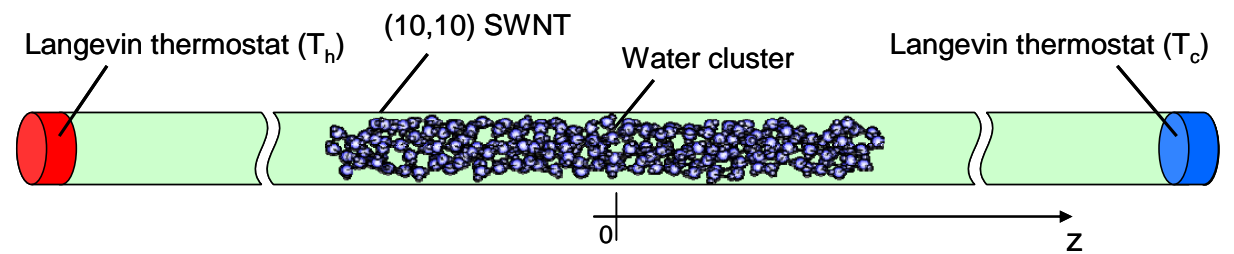

Fig. 5 A schematic of the transport simulation. A cluster of 192 water molecules is placed inside a $64 \mathrm{~nm}$ long (10, 10) SWNT, and the temperature gradient is applied by heating and cooling the left and right ends, respectively, by Langevin thermostats [29]. 


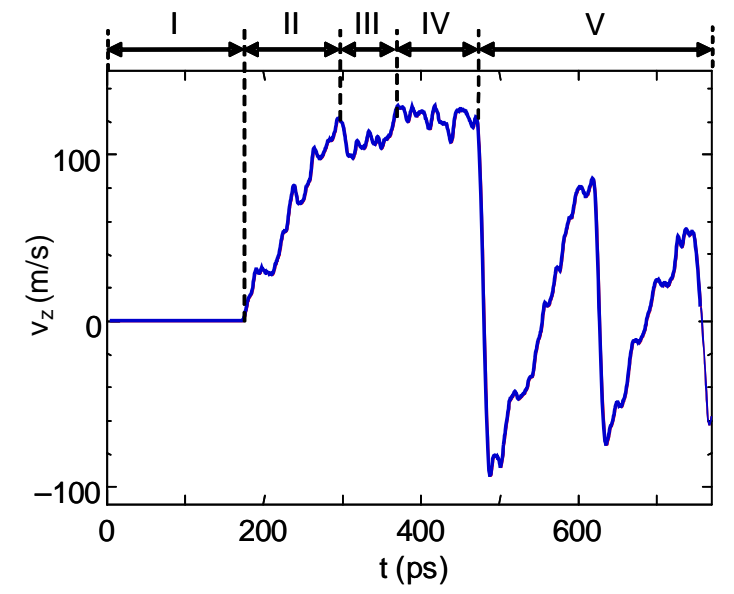

Fig. 6 The axial velocity time history $v_{z}(t)$ of the mass center of the water cluster. The imposed temperature gradient is $1.48 \mathrm{~K} / \mathrm{nm}$. The time history is divided into, (I) initial phase with $v_{z}=0$, (II) inertial phase with constant acceleration, (III) transition phase, (IV) steady phase with zero acceleration, and (V) the end phase with the cluster oscillating at the tube end.

could relate the temperature dependence of the potential energy and the force experienced by the water cluster. In case of water cluster inside a carbon nanotube, due to the hydrophobicity, it is expected that the water-water interaction plays dominant role compared with the water-carbon interaction, and therefore the intrinsic energetics may become more important than the interfacial ones.

\section{Influence of structural defects on water transport}

The influence of a junction of nanotubes with different diameters was also investigated. As shown in Fig. 7, a $(9,9)$ SWNT and a $(8,8)$ SWNT were smoothly connected using 5membered and 7-membered rings at the junction. In the same manner as the previous simulations for the smooth $(10,10)$ SWNT, the water transport simulations were performed by heating/cooling the end of the $(9,9) /(8,8)$ SWNT. The temperature gradient here is $1.48 \mathrm{~K} / \mathrm{nm}$. In this case, the water cluster was initially placed off center at around $z=-6 \mathrm{~nm}$, inside the $(9,9)$ SWNT. After releasing the water cluster, the water cluster was transported towards the cold side i.e. towards with junction at a speed of approximately $90 \mathrm{~m} / \mathrm{s}$, similar to the value of the smooth $(10,10)$ SWNT case for the same temperature gradient. However, in this case, the cluster never crosses the junction, as shown in the trajectory and the sequent pictures (Fig. 7). Instead, when the water cluster reaches the junction, it oscillates due to the potential barrier at the junction counteracting the thrust from the temperature gradient. This is due to the fact that the water cluster is more stable inside the (9, 9) SWNT than inside the $(8,8)$ SWNT. Such difference has been already seen in the previous work [33], which showed that slight change in the diameter results in a significant variation of the potential energy and molecular structure. In the current case, the cluster does not flow into the $(8,8)$ SWNT, since the temperature gradient effect cannot overcome the potential energy barrier caused by the diameter difference, with the current temperature gradient. The current results suggest that a nanotube junction, often seen in synthesized SWNTs, may become a key obstacle. It should also be noted that such effect is expected to be particularly pronounce in the current small diameter SWNTs, and the influence is expected to become weaker as the diameter increases.

\section{CONCLUSIONS}

Molecular dynamic simulations were performed to characterize the heat and mass transport in carbon nanotube systems. The results demonstrate unique nonequilibrium features that arise from the dominant surface area in singlewalled carbon nanotube. By adopting the spectral analyses on the non-stationary molecular dynamics simulations of SWNT/argon interface, we have identified the contribution of elastic and inelastic energy transport on the thermal boundary
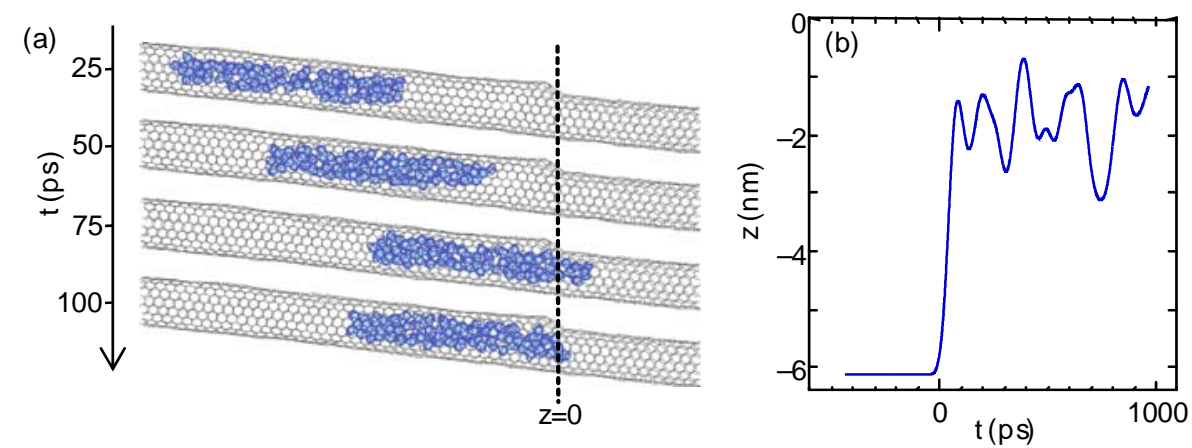

Fig. 7 The water cluster transport simulations with the $(9,9)-(8,8)$ SWNT junction. A temperature gradient of $1.48 \mathrm{~K} / \mathrm{nm}$ was applied to drive the water cluster in the $(9,9)$ SWNT towards the junction; (a) the time sequence images of the water cluster during the transport, and (b) the time history of the water cluster center of mass. The water cluster was initially $(\mathrm{t}<0)$ placed at $\mathrm{z}=-6 \mathrm{~nm}$. 
conductance. It is shown that the elastic energy transport is much faster than the inelastic one, but carries much smaller energy due to slow intra-SWNT phonon relaxation. This indicates that the quasi-ballistic phonon transport in SWNT not only characterizes the heat conduction along SWNTs but also influences the thermal boundary resistance between SWNTs and the surrounding materials.

The molecular dynamics simulations also demonstrate that carbon nanotubes are efficient mass transporter when water is confined inside. It is shown that, by applying the temperature gradient along the nanotube, the confined water can be efficiently transported at an average acceleration proportional to the temperature gradient. However, the transport simulations with a junction of two different SWNTs suggest that an angstrom diameter difference may result in a significant drag for small diameter SWNTs.

\section{ACKNOWLEDGMENTS}

The works presented here have been support in part by the Japan Society for the Promotion of Science.

\section{REFERENCES}

1. R. Saito, G. Dresselhaus, and M. S. Dresselhaus: Physical Properties of Carbon Nanotubes (Imperial College Press, London, 1998).

2. J. Hone, M. C. Llaguno, M. J. Biercuk, A. T. Johnson, B. Batlogg, Z. Benes, and J. E. Fischer: Appl. Phys. A 74 (2002) 339.

3. P. Kim, L. Shi, A. Majumdar, and P. L. McEuen, Phys. Rev. Lett. 87 (2001) 215502.

4. M. Fujii, X. Zhang, H. Xie, H. Ago, K. Takahashi, T. Ikuta, H. Abe, and T. Shimizu, Phys. Rev. Lett. 95 (2005) 065502 .

5. C. Yu, L. Shi, Z. Yao, D. Li, and A. Majumdar, Nano Lett. 5 (2005) 1842.

6. E. Pop, D. Mann, Q. Wang, K. Goodson, and H. Dai, Nano Lett. 6 (2006) 96.

7. T.-Y. Choi, D. Poulikakos, J.Tharian, and U. Sennhauser, Nano Lett., 6 (2006) 1589.

8. S. Maruyama, Physica B 323 (2002) 193.

9. S. Maruyama, Nanoscale Microscale Thermophys. Eng. 7 (2003) 41.

10. R. Livi and S. Lepri, Nature 421 (2003) 327.
11. N. Mingo and D. A. Broido, Nano Lett. 5 (2005) 1221.

12. J. Wang and J.-S. Wang, Appl. Phys. Lett. 88 (2006) 111909.

13. C. W. Chang, D. Okawa, H. Garcia, A. Majumdar, and A. Zettl, Phys. Rev. Lett. 101 (2008) 075903.

14. J. Shiomi and S. Maruyama, Phys. Rev. B 73 (2006) 205420

15. J. Shiomi and M. Maruyama, Jpn. J. Appl. Phys. 47 (2008) 2005.

16. M. J. Biercuk, etal., Appl. Phys. Lett. 80 (2002) 2767.

17. S. U. S. Choi, etal., Appl. Phys. Lett. 79 (2001) 2252.

18. S. T. Huxtable, et al., Nature Materials 2 (2003) 731.

19. S. Shenogin, etal., J. Appl. Phys. 95 (2004) 8136.

20. C. F. Carlborg, J. Shiomi, and S. Maruyama, Phys. Rev. B 78 (2008) 205406.

21. Y. Maniwa, H. Kataura, M. Abe, A. Udaka, S. Suzuki, Y. Achiba, H. Kira, K. Matsuda, H. Kadowaki, and Y. Okabe, Chem. Phys. Lett. 401 (2005) 534.

22. I. Kolesnikov, J.-M. Zanotti, C.-K. Loong, P. Thiyagarajan, A. P. Moravsky, R. O. Loutfy, and C. J. Burnham, Phys. Rev. Lett. 93 (2004) 035503.

23. N. Naguib, H. Ye, Y. Gogotsi, A. G. Yazicioglu, C. M. Megaridis, and M. Yoshimura, Nano Lett. 4 (2004) 2237.

24. M. Majumder, N. Chopra, R. Andrews, and B. J. Hinds, Nature London 438 (2005) 44.

25. J. K. Holt, H. G. Park, Y. Wang, M. Stadermann, A. B. Artyukhin, C. P. Grigoropoulos, A. Noy, and O. Bakajin, Science 312 (2006) 1034.

26. J. A. Thomas and A. J. H. McGaughey, Nano Lett. 8 (2008) 2788.

27. H. A. Zambrano, J. H. Walther, Pe. Koumoutsakos and I. F. Sbalzarini, Nano Letters 9 (2009) 66.

28. J. Shiomi and S. Maruyama, Nanotechnology 20 (2009) 055708 .

29. D. W. Brenner: Phys. Rev. B 42 (1990) 9458.

30. H. J. C. Berendsen, J. R. Grigera, and T. P. J. Straatsma, J. Phys. Chem. 91 (1987) 6269.

31. P. A. E. Schoen, J. H. Walther, S. Arcidiacono, D. Poulikakos and P. Koumoutsakos, Nano Lett. 6 (2006) 1910.

32. P. A. E. Schoen, J. H. Walther, D. Poulikakos and P. Koumoutsakos, Appl. Phys. Lett. 90 (2007) 253116.

33. J. Shiomi, T. Kimura, and S. Maruyama, J. Phys. Chem. C 111 (2007) 12188. 